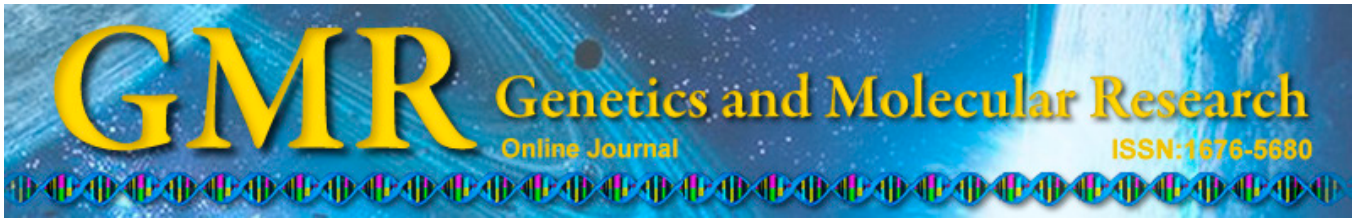

\title{
Molecular cloning, characterization, and expression analysis of a Broad-Complex homolog during development in the oriental river prawn Macrobrachium nipponense
}

\author{
S.F. Jiang ${ }^{1}$, Y.P. Zhang ${ }^{2}$, S.M. Sun ${ }^{1}$, Y.S. Gong ${ }^{1}$, Y.W. Xiong ${ }^{1}$, H. Qiao ${ }^{1}$, \\ W.Y. Zhang ${ }^{1}$, S.B. Jin ${ }^{1}$ and H.T. Fu ${ }^{1}$ \\ ${ }^{1}$ Key Laboratory of Freshwater Fisheries and Germplasm Resources Utilization, \\ Ministry of Agriculture, Freshwater Fisheries Research Center, \\ Chinese Academy of Fishery Sciences, Wuxi, China \\ ${ }^{2}$ Jiangxi Fisheries Research Institute, Nanchang, China \\ Corresponding author: H.T. Fu \\ E-mail: fuht@ffrc.cn
}

Genet. Mol. Res. 14 (2): 5141-5152 (2015)

Received May 20, 2014

Accepted November 5, 2014

Published May 18, 2015

DOI http://dx.doi.org/10.4238/2015.May.18.4

\begin{abstract}
Broad-Complex $(B R-C)$ is an early ecdysone-responsive gene encoding a family of zinc-finger transcription factors. In this study, we isolated the full-length cDNA of a $B R-C$ homolog from the testes of the oriental river prawn (Macrobrachium nipponense), according to established expressed sequence tag information, using the rapid amplification of cDNA ends technique. The homolog was designated as $M n B R-C$. The full-length cDNA of $M n B R-C$ contained a 1095-bp open reading frame encoding a precursor protein of 365 amino acid residues. Comparative and bioinformatic analyses revealed that $M n B R-C$ exhibited a high degree of homology with BR-C proteins, and contained the BTB and $\mathrm{Zf}-\mathrm{H} 2 \mathrm{C} 2-2$ domains. Real-time quantitative polymerase chain reaction (qPCR) analysis revealed that the $M n B R-C$ expression level varied significantly in the developing embryo, postembryonic
\end{abstract}


larva, and adult tissue. Real-time qPCR showed that the $M n B R-C$ gene was expressed in all of the tissues investigated, with the highest level of expression in the brain. In addition, $M n B R-C$ was more abundantly expressed in the testes than in the ovaries.

Key words: Macrobrachium nipponense; Broad-Complex; cDNA; Real-time quantitative polymerase chain reaction; Metamorphosis

\section{INTRODUCTION}

Broad-Complex $(B R-C)$ is a primary response gene in the ecdysteroid signaling cascade that encodes a family of $\mathrm{C} 2 \mathrm{H} 2$ zinc-finger transcription factors, which are involved in a number of processes in insects, such as oogenesis, vitellogenesis, and metamorphosis (Deng and Bownes, 1997; Chen et al., 2004; Dubrovsky, 2005; Spokony and Restifo, 2007). In Blattella germanica, $B R-C$ factors appear to be involved in cell division and wing pad growth (Huang et al., 2013).

In the fruit fly Drosophila melanogaster, $B R-C$ is one of the early genes, and plays key roles in both coordinating the ecdysone response among tissues and in selective activation or silencing of the downstream effecter genes (Thummel, 2002). BR-C proteins have a common core region fused to one of four different $\mathrm{C} 2 \mathrm{H} 2$ zinc-finger motifs, and generate four classes of protein (Z1, Z2, Z3, and Z4 isoforms) by alternative splicing (DiBello et al., 1991; Bayer et al., 1996). $B R-C$ coordinates early metamorphic events (Karim et al., 1993). BR-C mutants result in lethality during larval, prepupal, and pupal development, consistent with $B R-C$ playing a key role during metamorphosis (Stewart et al., 1972; Kiss et al., 1976; Belyaeva et al., 1980). These mutants die as wandering larvae, unable to enter metamorphosis and initiate pupariation. Cloning and expression analysis of the $B R-C$ gene has been studied in many species, such as the dipteran D. melanogaster, the lepidopterans Bombyx mori and Manduca sexta, the coleopteran Tribolium castaneum, and the crustacean Penaeus monodon (Uhlirova et al., 2003; Nishita and Takiya, 2004; Yoshinori and Shigeharu, 2004; Konopova and Jindra, 2008; Suzuki et al., 2008; Konopova et al., 2011; Buaklin et al., 2013).

We found a homologous sequence fragment of $B R-C$ from the testes cDNA library of Macrobrachium nipponense (Crustacea, Decapoda, Palaemonidae), which is a commercial freshwater prawn (Qiao et al., 2012). It is considered an important fishery resource in China, with an annual production of 205,010 tons (Bureau of Fishery, 2009). The molecular mechanisms regulating the expression of development genes in $M$. nipponense have received a great deal of attention recently (Zhang et al., 2013a, b, c), and it is possible that $B R-C$ may provide a link between pattern formation and cell behavior in morphogenesis. In the present study, we aimed to obtain a full cDNA clone encoding the $M n B R-C$ gene from the cDNA library of the testes of $M$. nipponense, and to examine the expression pattern of the $M n B R-C$ gene during embryonic development, larval stages, and post-larval stages.

\section{MATERIAL AND METHODS}

\section{Embryo, larvae, and tissue collections}

Several healthy adult oriental river prawns with wet weights of between 1.26 and 
4.25 g were obtained from Tai Lake, Wuxi, China $\left(120^{\circ} 13^{\prime} 44^{\prime \prime} \mathrm{E}, 31^{\circ} 28^{\prime} 22^{\prime \prime} \mathrm{N}\right)$. The samples were transferred to laboratory breeding conditions and maintained in a 500-L tank with aerated freshwater for $72 \mathrm{~h}$ before tissue collection. The different developmental stages of the eggs and larvae were obtained from our breeding room. After spawning, each developmental stage of the embryos [cleavage stage (CS), blastula stage (BS), gastrula stage (GS), nauplius stage (NS), protozoea stage (PS), and zoea stage (ZS)] was collected following the criteria of Chen et al. (2012). Larvae were collected every 3 days, between 1 day post-hatching (L1) and L13 (a day before metamorphosis). Post-larvae were collected every 4 days, between 1 and 20 days after metamorphosis (P1-P20), and every 10 days between P20 and P30. Several tissues, including the ovaries, testes, muscle, heart, abdominal ganglion, and brain were also collected. The samples were washed with $1 \mathrm{X}$ phosphate-buffered saline $(0.01 \mathrm{M})$, frozen directly in liquid nitrogen, and stored at $-80^{\circ} \mathrm{C}$ until being processed.

\section{RNA isolation and reverse transcription}

Total RNA was extracted from the embryos and larvae at different stages of development, and from other tissues in mature prawns, using RNAiso Plus reagent (TaKaRa Bio Inc., Japan) in accordance with the protocol of the manufacturer. The isolated RNA was treated with RNase-free DNase I (Sangon, Shanghai, China) to eliminate possible genomic DNA contamination. The concentration of each total RNA sample was then measured using a BioPhotometer (Eppendorf, Hamburg, Germany), and $2 \mu \mathrm{L}$ was analyzed on a $1 \%$ agarose gel to check its integrity. The cDNA was synthesized from $5 \mu \mathrm{g}$ total RNA using a PrimeScript ${ }^{\mathrm{TM}}$ RT-PCR Kit (TaKaRa Bio Inc.) according to the manufacturer protocol. The cDNA was kept at $-20^{\circ} \mathrm{C}$ for real-time quantitative reverse transcription-polymerase chain reaction (RT-PCR) analysis.

\section{5'- and 3'-rapid amplification of cDNA ends (RACE) amplification of the $B R$ - $C$ gene}

Four gene-specific primer sets (Table 1) were designed based on the expressed sequence tag (EST) of a $B R-C$ homolog (GenBank Accession No. JK527173) obtained from the M. nipponense testes cDNA library (Qiao et al., 2012). The cloning of $M n B R-C$ cDNA and 3'- and 5'-RACE cDNA was performed using the 3'-full RACE Core Set Ver. 2.0 (TaKaRa Bio Inc.) and the 5'-full RACE Kit (TaKaRa Bio Inc.), respectively, according to manufacturer instructions. For the 5'-RACE, the primer sets consisted of two genespecific primers of GSP1 and GSP2 and the universal primers 5' RACE OUT and 5' RACE IN (Table 1). For the 3'-RACE, the primer sets consisted of two gene-specific primers of GSP3 and GSP4 and the universal primers 3' RACE OUT and 3' RACE IN (Table 1).

The PCR products were gel-purified and ligated into a pMD18-T vector (TaKaRa Bio Inc.) following the instructions provided by the manufacturer. The recombination was then transformed into Escherichia coli DH5a (Qiagen, Germany) competent cells, which were identified by blue/white screening, and confirmed by PCR. Five of the positive clones were sequenced in both directions using an automatic DNA sequencer (Model 3730, Applied Biosystems, Shanghai, China) and the resulting sequences were verified and subjected to cluster analysis using the online database of the National Center for Biotechnology Information (NCBI). 
Table 1. Nucleotide sequences of primers used for the characterization and expression analysis of $M n B R-C$.

\begin{tabular}{ll}
\hline Primer & Primer sequence $\left(5^{\prime}-3^{\prime}\right)$ \\
\hline BR-CF & GGGGCCTAGCTGTACCTGAT \\
BR-CR & CTGCCGGAACATCACTGGAG \\
BR-C-3GSP1 & AAAAGCCCCATCAGACGA \\
BR-C-3GSP2 & GCACTAAAACGCCAACCC \\
BR-C-5GSP1 & GGCTGTCGTGTCTTTCGTCTGATG \\
BR-C-5GSP2 & CCAAGAGCGCATCCATATCCTGAC \\
3' RACE OUT & TACCGTCGTTCCACTAGTGATTT \\
3' RACE IN & CGCGGATCCTCCACTAGTGATTTCACTATAGG \\
5 $^{\prime}$ RACE OUT & CATGGCTACATGCTGACAGCCTA \\
5 RACE IN $^{\prime}$ R-CRTF & CGCGGATCCACAGCCTACTGATGATCAGTCGATG \\
BR-CRTR & GCCATCCCCAGACCACTTAG \\
$\beta$-actinF & TCATGATCGTGTTCTTGCATTTC \\
$\beta$-actinR & TATGCACTTCCTCATGCCATC \\
\hline
\end{tabular}

\section{Nucleotide sequence and bioinformatic analyses}

The $5^{\prime}$ and $3^{\prime}$ sequences from the RACEs were assembled with the partial cDNA sequence corresponding to each fragmental sequence using DNAMAN 5.0. Sequences were analyzed based on the nucleotide and protein databases using the BLASTx and BLASTn programs (http://blast.ncbi.nlm.nih.gov/Blast.cgi). Protein prediction was performed using an open reading frame (ORF) finder (http://www.ncbi.nlm.nih.gov/gorf/). The ProtParam program (http://web.expasy.org/protparam/) was used to compute physical and chemical parameters of the amino acid sequence. The motif was searched with the motif scan program (http:// myhits.isb-sib.ch/cgi-bin/motif_scan). Multiple alignments of $B R-C$ (Table 1) were generated using the Clustal W 1.81 program (http://www.clustal.org/clustal2/), and the obtained alignments were used to construct phylogenetic trees by the neighbor-joining (NJ) method. A bootstrap test was then performed with 1000 replications, using MEGA 4 (Molecular Evolutionary Genetics Analysis; Tamura et al., 2007).

\section{Real-time qPCR analysis of $B R-C$}

$B R-C$ mRNA expression at different stages, from embryo to post-larval, and in various adult tissues, was measured using a SYBR ${ }^{\circledR}$ Green Real-Time qRT-PCR Kit and a Bio-Rad iCycler iQ5 Real-Time System (Bio-Rad, Hercules, CA, USA). Gene-specific primers (Table 1) were used to amplify the $M n B R-C$ transcript, and the PCR products were sequenced to verify the specificity of the PCR primers. Three samples were collected from each developmental stage and each tissue. Each sample was performed in triplicate, with each reaction well containing a $25-\mu \mathrm{L}$ PCR mixture composed of $1 \mu \mathrm{L}$ cDNA (50 ng), $10 \mu \mathrm{L}$ SsoFast $^{\mathrm{TM}}$ EvaGreen $^{\circledR}$ Supermix (Bio-Rad), $0.5 \mu \mathrm{L} 10 \mu \mathrm{M}$ of gene-specific forward and reverse primers (Table 1), and $13 \mu \mathrm{L}$ diethyl pyrocarbonate (DEPC) water. The reaction mixture was initially incubated for $30 \mathrm{~s}$ at $95^{\circ} \mathrm{C}$ to activate the Hot Start Taq DNA polymerase (Takara, Japan), followed by 40 cycles of $10 \mathrm{~s}$ denaturation at $95^{\circ} \mathrm{C}$ and $10 \mathrm{~s}$ extension at $60^{\circ} \mathrm{C}$. A melt curve analysis was performed over a temperature range of $65-95^{\circ} \mathrm{C}$ (in $0.5^{\circ} \mathrm{C}$ increments) for $10 \mathrm{~s}$, to verify the single-product generation at the end of the assay. The amplification of $\beta$-actin as an internal reference was also carried out in the same sample (the primer's sequences are shown in Table 1). DEPC water for the 
replacement of the template was used as a negative control. The relative copy number of $M n B R-C$ mRNA was calculated according to the $2^{-\Delta \Delta C T}$ comparative CT method (Livak and Schmittgen, 2001).

\section{Statistical analysis}

All data are reported as means $\pm \mathrm{SE}$ (standard error of the mean; $\mathrm{N}=3$ ). Statistical analysis was performed using the SPSS 13.0 software, and statistical significance was determined using one-way analysis of variance and post hoc Duncan multiple range tests. Significance was set at $\mathrm{P}<0.05$.

\section{RESULTS}

\section{Analysis of the $M n B R-C$ nucleotide sequence}

The cloning of $B R-C$ cDNAs in M. nipponense was accomplished by a RT-PCR approach using specific primers designed based on the EST. The full-length cDNA sequence of $M n B R-C$ was determined by merging the sequences of the 3'- and 5'-RACE products. A BLAST search revealed that it is homologous to the $B R-C$ gene. The nucleotide identity between this protein and the $B R-C$ gene of $P$. monodon (JN638741.1) was $71 \%$. The full-length cDNA was comprised of $1566 \mathrm{bp}$, including $1095 \mathrm{bp}$ in the ORF, $178 \mathrm{bp}$ in the 5'-untranslated region, and $292 \mathrm{bp}$ in the 3'-untranslated region, with a poly (A) tail (Figure 1). The $M n B R-C$ cDNA sequence has been submitted to GenBank with the Accession No. KJ767635.

\section{Analysis of the $M n B R-C$ predicted protein sequence}

The analysis of the deduced protein sequence of $M n B R-C$ revealed that it was comprised of 365 amino acids, and had a predicted molecular mass of $40.67 \mathrm{kDa}$ and an isoelectric point of 5.62. The amino acid identity between this protein and that of the BR-C gene of P. monodon (AEU11366.1) was 58\%. BLAST searches indicated that it encoded a $M$. nipponense ortholog of $B R-C$, which contained conserved-protein domains of the BTB and $\mathrm{Znf}-\mathrm{H} 2 \mathrm{C} 2 \_2$ domains. Therefore, we named it MnBR-C. The protein functional sites of $M n B R-C$ were speculated using ExPASy (http://www.expasy.org/). The result show that this protein is composed of seven protein kinase $\mathrm{C}$ phosphorylation sites, six casein kinase II phosphorylation sites, two tyrosine kinase phosphorylation sites, three $\mathrm{N}$-myristoylation sites, and one N-glycosylation site. These results indicate that the MnBR-C protein has multiple potential functional sites for phosphorylation, glycosylation, and methylation.

\section{Homology and phylogenetic analysis of $M n B R-C$}

Multi-alignment analysis by Clustal $\mathrm{W}$ indicated that $M n B R-C$ is similar to the $B R-C$ reported in other species, sharing 58\% identity with P. monodon (AEU11366.1) and 44\% identity with Acromyrmex echinatior (EGI63786.1) (Figure 2). 


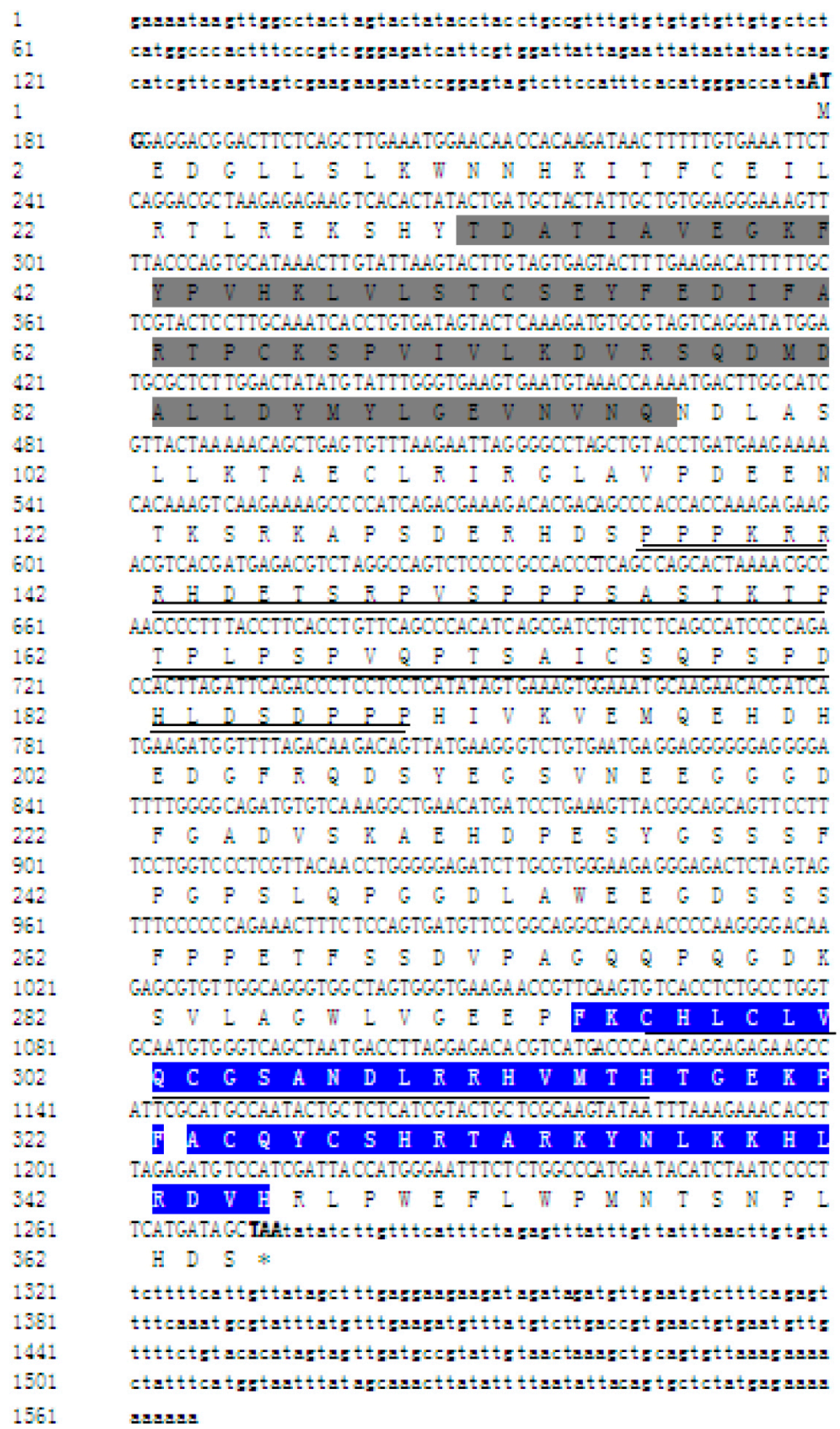

Figure 1. Nucleotide and deduced amino acid sequences of $M n B R-C$ from oriental river prawns (Macrobrachium nipponense). An asterisk denotes the termination codon. The nucleotide sequence is displayed in the 5'-3' direction and numbered to the left. The deduced amino acid sequence is shown in a single capital letter amino acid code. $3^{\prime}$ UTR and 5' UTR are listed with lowercase letters. BTB and two zinc-finger C2H2-type domains are marked with gray or blue shadows, respectively. The Proline-rich region is marked with a double line, and the zinc-finger $\mathrm{C} 2 \mathrm{H} 2$ type domain signature is underlined. 
embryos at different developmental stages, and on larval and post-larval stages. The RT-qPCR analysis revealed that the $M n B R-C$ expression level changed significantly from the developmental stages of the embryo to the post-larval stages $(\mathrm{P}<0.05)$. At the early embryonic developmental stage, the expression level of MnBR-C was higher at the CS (353.67-fold) than at the BS (10.62-fold). As the embryo developed, $M n B R-C$ mRNA was expressed at a low level, but gradually increased from the GS (14.8-fold) to the PS (48.56-fold), and then abruptly decreased at the ZS (9.09-fold) (Figure 4). MnBR-C expression was maintained at a low level during all of the larval stages (Figure 4). After metamorphosis, the larvae transitioned into post-larvae that resembled miniature adults. During the post-larval stage, the highest expression was observed at P1 (705.97-fold), which gradually decreased from P5 (93.26-fold) to P30 (20.01-fold) (Figure 4).

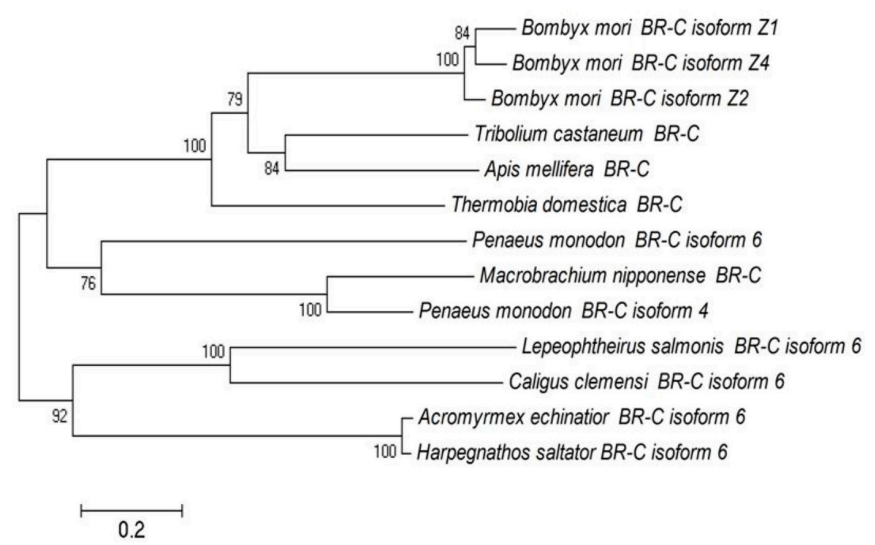

Figure 3. Neighbor-joining phylogenetic tree based on $B R-C$ sequences from different species.

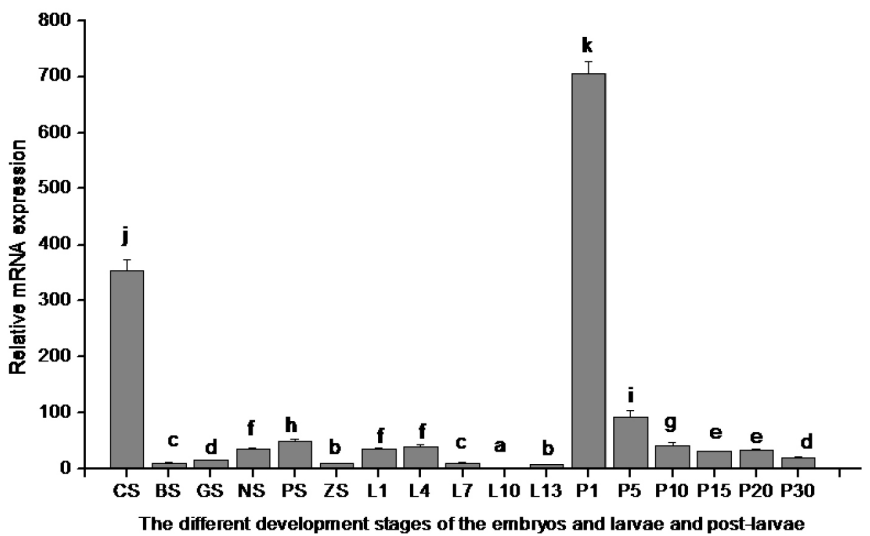

Figure 4. Temporal expression of a Broad-Complex $(B R-C)$ homolog during the different development stages of embryo, larva before metamorphosis and larva after metamorphosis, was revealed using real-time quantitative polymerase chain reaction analysis. The amount of $B R-C$ mRNA was normalized to the $\beta$-actin transcript level. Data are reported as means \pm SD (standard deviation) of three repeated samples during embryonic, larval, and post-larval stages. Bars with different letters were considered to be significant at $\mathrm{P}<0.05$. CS, cleavage stage; BS, blastula stage; GS, gastrula stage; NS, nauplius stage; PS, protozoea stage; ZS, zoea stage. L1, the first day after hatching; P1, the first day after metamorphosis. 


\section{Expression analysis of MnBR-C mRNA in various tissues}

RT-qPCR analysis revealed that the $M n B R-C$ gene was expressed in all of the tissues investigated, with the highest mRNA levels observed in the brain (192.14-fold), followed by the testes $(62.68$-fold), and the ovaries $(10.13$-fold). The lowest levels were detected in the abdominal ganglion (1.3-fold) and the heart (the calibrator) (Figure 5).

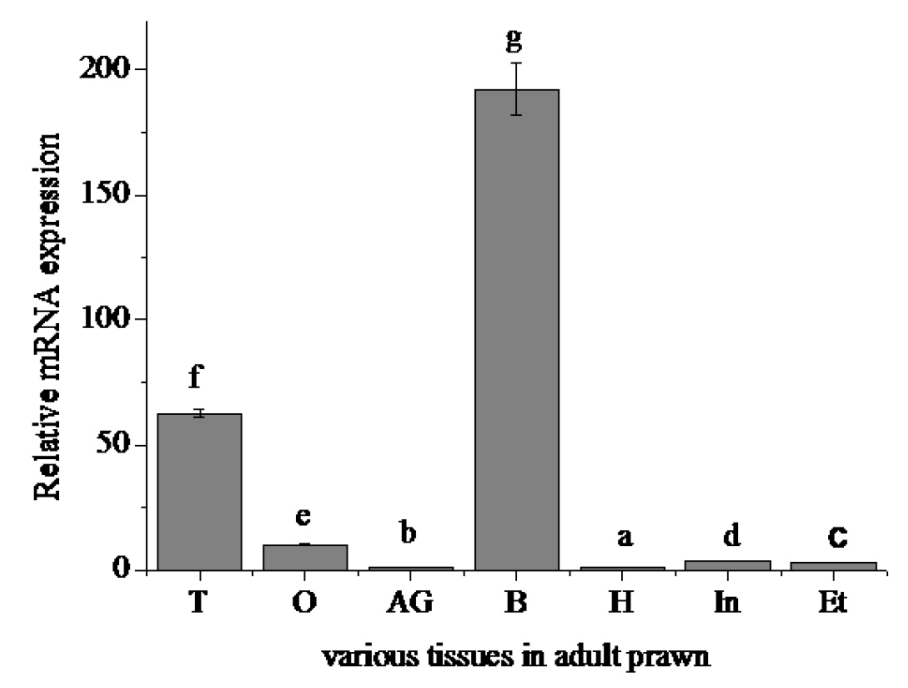

Figure 5. Relative expression levels of $M n B R-C$ transcripts quantified in various adult tissues. $T$, testes; $\mathrm{O}$, ovaries; AG, abdominal ganglion; B, brain; $\mathrm{H}$, heart; In, intestine; Et, eyestalk.

\section{DISCUSSION}

BR-C proteins consist of an N-terminal BTB (bric-a-brac, tramtrack, and $B R-C$ ) domain, followed by a central region of several hundred residues, and ending with a series of $\mathrm{C} 2 \mathrm{H} 2$-type zinc-finger domains at the $\mathrm{C}$-terminus, generating four classes of proteins, the $\mathrm{Z} 1$, Z2, Z3, and Z4 isoforms, by alternative splicing (DiBello et al., 1991; Bayer et al., 1996; Zhou et al., 1998). The N-terminus of the core region is highly conserved, serves in protein-protein interactions, and is referred to as the BTB. The BTB domain is characteristic of the GAGA factor and other chromatin-altering factors (Albagli et al., 1995). In this study, we successfully cloned the full-length cDNA of the $B R-C$ gene based on the EST from a $M$. nipponense testes cDNA library. The cDNA sequence encoded a protein of 365 amino acids having a molecular weight of $40.67 \mathrm{kDa}$. Amino acid sequence analysis revealed that $M n B R-C$ shares a high homology with the previously reported crustacean P. monodon BR-C (PmBR-C) proteins, but it exhibited low sequence similarity levels with the previously characterized $B R-C$ of other species (data not shown). A high degree of similarity was observed in the BTB domain of $M n B R-$ $C$ with other insects. This conservation suggests that $M n B R-C$ probably performs conserved functions as a GAGA factor, and other chromatin-altering factors. Phylogenetic analysis revealed that $M n B R-C$ was closely related to the crustacean $P m B R-C$ isoform 4 and isoform 6 , and separated from its homologs in insects. The precise orthologous relationships of $M n B R-C$ 
need further study, after further $B R$ - $C s$ are cloned from crustaceans.

It has been reported that $B R-C$ pre-mRNA can produce multiple alternatively spliced mRNAs that encode multiple isoforms of the BR-C protein via an alternative splicing pathway in some species, such as in P. monodon and B. germanica (Buaklin et al., 2013; Huang et al., 2013). We only cloned a single $B R-C$ mRNA in this study; to thoroughly understand the biological function of $B R-C$ in crustaceans, further studies should be focused on studying the $B R-C$ DNA sequence and splicing pathway during processing in crustaceans.

The results of previous studies have indicated that the BR-C protein is widely distributed throughout the tissues, from the late larval to the prepupal stages of development (Bayer et al., 1996; Zhou and Riddiford, 2002; Reza et al., 2004). The expression of $B R$ - $C$ throughout the second half of embryonic development in D. melanogaster (Sullivan and Thummel, 2003) would suggest a possible function in the embryo. In the present study, our RT-qPCR results show that $M n B R-C$ mRNA was also expressed at all developmental stages in $M$. nipponense, including the embryonic, larval, and post-larval stages. A high level of $M n B R-C$ mRNA was expressed at the CS of M. nipponense embryos, but decreased at the BS and stayed at a low level from the BS to the GS, suggesting that there is a maternal deposit in embryos (Piulachs et al., 2010). As the embryo developed, $M n B R-C$ mRNA gradually increased from the GS (14.8fold) to the PS (48.56-fold), and then abruptly decreased at the ZS, suggesting that MnBR-C participates in the embryonic development of $M$. nipponense.

In Aedes aegypti, M. sexta, and B. mori, expression studies have confirmed that $B R-C$ expression is ecdysteroid-dependent (Zhou et al., 1998; Chen et al., 2004; Ijiro et al., 2004; Nishita and Takiya, 2004; Reza et al., 2004). Metamorphosis is one of the most emblematic processes regulated by $B R-C$ (Karim et al., 1993; Zhou et al., 1998). M. nipponense larvae need to undergo the molting process, which accompanies changes in morphology, many times. During these periods, the $M n B R-C$ level gradually increased at a late larval stage of premetamorphosis and reached its highest level during metamorphosis. After metamorphosis, $M n B R-C$ expression abruptly decreased. Therefore, we suggest that $M n B R-C$ mRNA may play a role in early metamorphosis.

Tissue expression analyses are used to determine the spatial distributions and expression levels of $B R-C$ in adult tissues. In $P$. monodon, tissue distribution analysis revealed that $P m B R-C$ mRNA was abundantly expressed in the ovaries, and was expressed at a lower level in hemocytes, the gills, the heart, the lymphoid organs, the intestine, the stomach, the hepatopancreas, the thoracic ganglion, the pleopods, the antennal gland, and the testes, and also revealed a preferential PmBR-C expression in the ovaries rather than the testes, in both juveniles and broodstock, suggesting it plays more important roles in the ovarian rather than the testicular development of P. monodon (Bauklin et al., 2013). However, PmBR-C mRNA is not expressed in the eyestalks of broodstock (Bauklin et al., 2013). In this study, expression analysis in seven tissues (including the eyestalk) indicated that $M n B R-C$ mRNA was widely distributed in the adult tissues of $M$. nipponense. In addition, we found that $M n B R-C$ mRNA was abundantly expressed in the brain and testes. Moreover, the expression level of $M n B R-C$ mRNA in the testes was significantly higher (6-fold) than that in the ovaries, in contrast to that observed in P. monodon. The results of several studies suggest that $B R-C$ isoforms may have distinct functions in D. melanogaster (Emery et al., 1994; Spokony and Restifo, 2007). The significant differences in $B R-C$ mRNA expression in certain tissues between $M$. nipponense and $P$. monodon may have been caused by species or developmental differences or by the physiological conditions prior to sampling. 
In the present study, the $M n B R-C$ gene was identified and characterized. The expression profile of this gene at all $M$. nipponense developmental stages suggests that it may play a role in the metamorphosis of $M$. nipponense. Further investigations of the molecular mechanisms of $M n B R-C$ in metamorphosis and ovarian and testicular development need to be undertaken, for the practical application of controlling the reproductive maturation of female $M$. nipponense in captivity.

\section{ACKNOWLEDGMENTS}

Research supported by the Jiangsu Provincial Natural Science Foundation for Young Scholars of China (Grant \#BK2012091), the National Science and Technology Supporting Program of the 12th Five-Year Plan of China (Grant \#2012BAD26B04), the Science and Technology Supporting Program of Jiangsu Province (Grant \# BE2012334), the Special Fund for Agro-Scientific Research in the Public Interest (\#201303056-6), a National Nonprofit Institute Research Grant from the Freshwater Fisheries Research Center, CAFS (\#2013JBFT07), and the "Three New Projects" of Jiangsu Province (\#D2013-6).

\section{REFERENCES}

Albagli O, Dhordain P, Deweindt C, Lecocq G, et al. (1995). The BTB/POZ domain: a new protein-protein interaction motif common to DNA- and actin-binding proteins. Cell Growth Differ. 6: 1193-1198.

Bayer CA, Holley B and Fristrom JW (1996). A switch in broad-complex zinc-finger isoform expression is regulated posttranscriptionally during the metamorphosis of Drosophila imaginal discs. Dev. Biol. 177: 1-14.

Belyaeva ES, Aizenzon MG, Semeshin VF, Kiss II, et al. (1980). Cytogenetic analysis of the 2B3-4-2B11 region of the X-chromosome of Drosophila melanogaster. Chromosoma 81: 281-306.

Buaklin A, Sittikankaew K, Khamnamtong B, Menasveta P, et al. (2013). Characterization and expression analysis of the Broad-complex (Br-c) gene of the giant tiger shrimp Penaeus monodon. Comp. Biochem. Physiol. B 164: 280-289.

Bureau of Fishery (2009). Fisheries economic statistics. In: China Fishery Yearbook. China Agricultural Press, Beijing, 236 pp.

Chen L, Zhu J, Sun G and Raikhel AS (2004). The early gene Broad is involved in the ecdysteroid hierarchy governing vitellogenesis of the mosquito Aedes aegypti. J. Mol. Endocrinol. 33: 743-761.

Chen Y, Zhu Q, Chen H, Zhu, XL, et al. (2012). The morphological and histological observation of embryonic development in the oriental river prawn Macrobrachium nipponense. J. Shanghai Ocean Univ. 21: 33-40.

Deng WM and Bownes M (1997). Two signalling pathways specify localised expression of the Broad-Complex in Drosophila eggshell patterning and morphogenesis. Development 124: 4639-4647.

DiBello PR, Withers DA, Bayer CA, Fristrom JW, et al. (1991). The Drosophila Broad-Complex encodes a family of related proteins containing zinc fingers. Genetics 129: 385-397.

Dubrovsky EB (2005). Hormonal cross talk in insect development. Trends Endocrinol. Metab. 16: 6-11.

Emery IF, Bedian V and Guild GM (1994). Differential expression of Broad-Complex transcription factors may forecast tissue-specific developmental fates during Drosophila metamorphosis. Development 120: 3275-3287.

Huang JH, Lozano J and Belles X (2013). Broad-complex functions in postembryonic development of the cockroach Blattella germanica shed new light on the evolution of insect metamorphosis. Biochim. Biophys. Acta 1830: 2178-2187.

Ijiro T, Urakawa H, Yasukochi Y, Takeda M, et al. (2004). cDNA cloning, gene structure, and expression of BroadComplex (BR-C) genes in the silk worm, Bombyx mori. Insect Biochem. Mol. Biol. 34: 963-969.

Karim FD, Guild GM and Thummel CS (1993). The Drosophila Broad-Complex plays a key role in controlling ecdysoneregulated gene expression at the onset of metamorphosis. Development 118: 977-988.

Kiss I, Bencze G, Fodor G, Szabad J, et al. (1976). Prepupa larval mosaics in Drosophila melanogaster. Nature 262:136-138.

Konopova B and Jindra M (2008). Broad-Complex acts downstream of Met in juvenile hormone signaling to coordinate primitive holometabolan metamorphosis. Development 135: 559-568.

Konopova B, Smykal V and Jindra M (2011). Common and distinct roles of juvenile hormone signaling genes in metamorphosis of holometabolous and hemimetabolous insects. PLoS One 6: e28728.

Livak KJ and Schmittgen TD (2001). Analysis of relative gene expression data using real-time quantitative PCR and the 
2 (-Delta Delta C (T)) Method. Methods 25: 402-408.

Nishita Y and Takiya S (2004). Structure and expression of the gene encoding a Broad-Complex homolog in the silkworm, Bombyx mori. Gene 339: 161-172.

Piulachs MD, Pagone V and Bellés X (2010). Key roles of the Broad-Complex gene in insect embryogenesis. Insect Biochem. Mol. Biol. 40: 468-475.

Qiao H, Fu HT, Jin SB, Wu Y, et al. (2012). Constructing and random sequencing analysis of normalized cDNA library of testis tissue from oriental river prawn (Macrobrachium nipponense). Comp. Biochem. Physiol. D 7: 268-276.

Reza AM, Kanamori Y, Shinoda T, Shimura S, et al. (2004). Hormonal control of a metamorphosis-specific transcriptional factor Broad-Complex in silkworm. Comp. Biochem. Physiol. B Biochem. Mol. Biol. 139: 753-761.

Spokony RF and Restifo LL (2007). Anciently duplicated Broad Complex exons have distinct temporal functions during tissue morphogenesis. Dev. Genes Evol. 217: 499-513.

Stewart JW, Sherman F, Jackson M, Fred LX, et al. (1972). Demonstration of the UAA ochre codon in bakers yeast by amino-acid replacements in iso-1-cytochrome c. J. Mol. Biol. 68: 83-96.

Sullivan AA and Thummel CS (2003). Temporal profiles of nuclear receptor gene expression reveal coordinate transcriptional responses during Drosophila development. Mol. Endocrinol. 17: 2125-2137.

Suzuki Y, Truman JW and Riddiford LM (2008). The role of Broad in the development of Tribolium castaneum: implications for the evolution of the holometabolous insect pupa. Development 135: 569-577.

Tamura K, Dudley J, Nei M and Kumar S (2007). MEGA4: Molecular Evolutionary Genetics Analysis (MEGA) software version 4.0. Mol. Biol. Evol. 24: 1596-1599.

Thummel CS (2002). Ecdysone-regulated puff genes 2000. Insect Biochem. Mol. Biol. 32: 113-120.

Uhlirova M, Foy BD, Beaty BJ, Olson KE, et al. (2003). Use of Sindbis virus-mediated RNA interference to demonstrate a conserved role of Broad-Complex in insect metamorphosis. Proc. Natl. Acad. Sci. U.S.A. 100: 15607-15612.

Yoshinori N and Shigeharu T (2004). Structure and expression of the gene encoding a Broad-Complex homolog in the silkworm, Bombyx mori. Gene 339: 161-172.

Zhang YP, Fu HT, Qiao H, Jin SB, et al. (2013a). Molecular cloning and expression analysis of transformer-2 gene during development in Macrobrachium nipponense (de Haan). J. World Aquacult. Soc. 44: 338-349.

Zhang YP, Jiang SF, Xiong YW, Sun SM, et al. (2013b). Molecular cloning and expression analysis of extra sex combs gene during development in Macrobrachium nipponense. Turk. J. Fish. Aquat. Sci. 3: 331-340.

Zhang YP, Qiao H, Zhang WY, Sun SM., et al. (2013c). Molecular cloning and expression analysis of two sex-lethal homolog genes during development in the oriental river prawn, Macrobrachium nipponense. Genet. Mol. Res. 12: 4698-4711.

Zhou B, Hiruma K, Shinoda T and Riddiford LM (1998). Juvenile hormone prevents ecdysteroid-induced expression of broad complex RNAs in the epidermis of the tobacco hornworm, Manduca sexta. Dev. Biol. 203: 233-244.

Zhou X and Riddiford LM (2002). Broad specifies pupal development and mediates the 'status quo' action of juvenile hormone on the pupal-adult transformation in Drosophila and Manduca. Development 129: 2259-2269. 